\title{
Design and Modeling of a Low-Power Multi-Channel Integrated Circuit for Infrared Gas Recognition
}

\author{
S. Sutula, C. Ferrer and F. Serra-Graells \\ stepan.sutula@imb-cnm.csic.es
}

\begin{abstract}
The design and high-level modeling of a multi-channel integrated circuit for infrared gas recognition suitable for low-power consumption are presented. The thermal compensation and independent programmability methods are used in all the main channel stages: sensor biasing, high-pass filtering and pre-amplification, blind cancellation, lock-in demodulation and A/D conversion. CMOS transistor subthreshold operation and circuit reuse techniques are widely applied in order to obtain a low-power and compact channel realization. Experimental results are presented for a $340 \mu \mathrm{m} \times 880 \mu \mathrm{m}$ channel integrated in $0.35 \mu \mathrm{m}$ CMOS technology reporting good agreement with electrical and higher level simulations. Keywords: Gas recognition, infrared, real-time, low-power, lock-in, CMOS.
\end{abstract}

\section{Introduction}

In recent years, an increasing demand for multi-channel processing systems has been observed in areas such as medicine, industry or home automation $[1,2,3]$. The large number of sensors present in these systems and their parallel processing have increased considerably the complexity of the electronic equipment needed for its appropriate functioning. In addition to this, a preference for a low-power consumption and reduced available area for portable applications have led to the use of very large scale integration (VLSI) technologies [4].

In the present case, the multi-channel system we deal with is an infrared (IR) gas analyzer. Its principle of operation is based on the spectroscopic gas

Preprint submitted to Microprocessors and Microsystems: embedded hardware designDecember 15, 2011

http://dx.doi.org/10.1016/j.micpro.2012.02.011 
recognition [5], which is done by means of passing an IR light beam through a chamber with the gas under test. An array of IR sensors and optical filters on the chamber's output measures the light power on different infrared frequencies. Due to the dependence of IR power absorption by the gas molecules on the light wavelength, we can determine the type of gas from its absorption spectrum. In other words, this process is similar to analyzing a fingerprint and identifying who its owner is.

This paper presents a multi-channel digital read-out integrated circuit for IR spectroscopic gas recognition, which exhibits a low-power consumption and compact area per channel. The proposed channel circuitry also allows the full CMOS integration of Hz-range filters. Furthermore, the independent and extensive programmability of each signal processing channel enables the compensation of the process deviations within the IR sensing array. In order to cover both the long (LWIR) and middle wave infrared (MWIR) spectroscopic ranges, the compatibility of different types of devices, like microbolometer [6] and PbSe photoconductive [7] sensors, is preserved.

\section{Gas Recognition System Overview}

The gas recognition system based on IR spectroscopy of Fig. 1 is a portable unit with a wired or wireless communication with an external data unit and user interface. This allows its functioning in a large variety of places where the people could have restricted access. Basically, the pulsed IR emitter illuminates the gas under test inside the optical chamber, and the mirrors implement a multi-path cell to magnify the gas absorption losses. Once IR light reaches the sensor head, is first split through a passive array of optical filters according to the interesting set of wavelength bins. Then, the array of IR thermal sensors translates the incoming light power into an equivalent change in resistance. Hence, the aim of the read-out integrated circuit (ROIC) is to supply a multi-channel interface between the IR thermal sensor array and the digital domain where the gas recognition is really performed. As part of the ROIC signal processing, each 


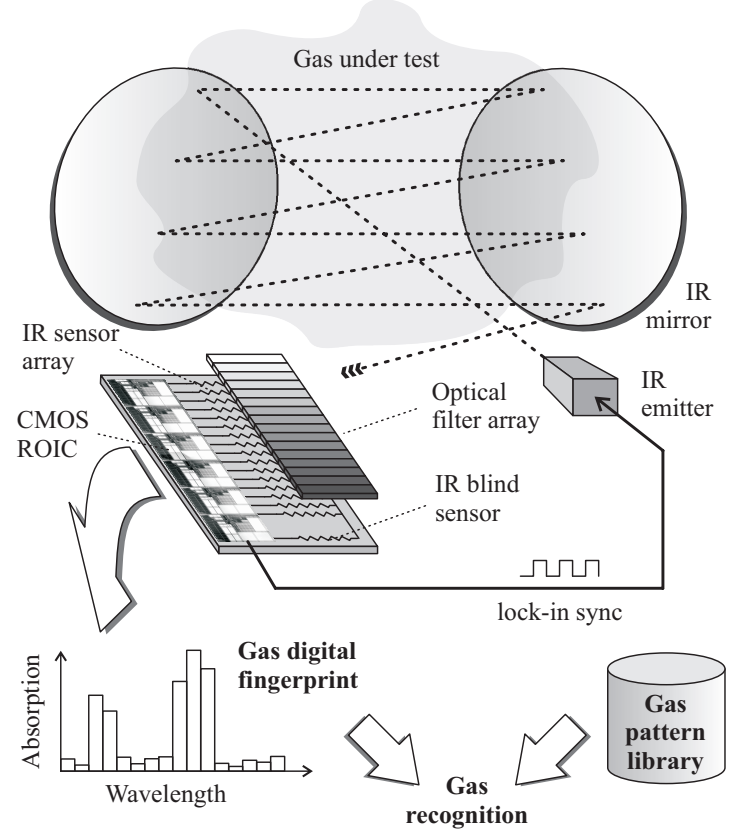

Figure 1: Infrared spectroscopic gas recognition system.

channel includes lock-in demodulation capabilities in phase with the IR emitter for improving the overall signal integrity. The minimum attenuation factor to be detected in the lock-in amplitude before and after the gas is inside the optical chamber depends on the minimum gas concentration and determines the dynamic range per channel.

\section{Read-Out Integrated Circuit Channel}

Each ROIC channel should meet the following specifications according to the general view of the gas recognition system of Fig. 1: built-in A/D conversion to minimize the overall noise bandwidth of the parallel processing system; no external components and reduced area to allow a compact packaging with the sensor array; high flexibility to be compatible with different IR sensor types and process deviations; and very low-power consumption to avoid any temperature drift close to the IR thermal sensors and to maximize the battery life in 
portable applications. For these purposes, the channel architecture of Fig. 2 is proposed, where $V_{\text {com }}$ is the bias common voltage for the whole IR sensor array. Each CMOS read-out channel consists of a sensor bias current source, a pre-amplifier with high-pass filtering capabilities, a linearized differential to single-ended transconductor for the blind channel common disturbance rejection, a pulse density modulator (PDM) followed by a digital first-order filter for the predictive A/D conversion and a digital serial I/O interface. Also, in order to achieve independent operation and to avoid crosstalk, all channels have their own built-in analog reference generator. Based on this scheme, each sensor is

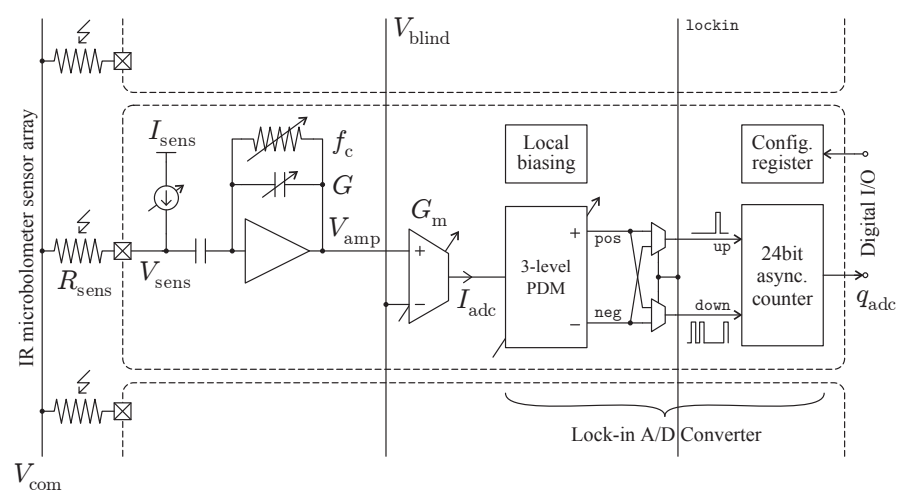

Figure 2: Functional description and programmability of the fully-integrable read-out channel.

biased separately by a constant but programmable current $\left(I_{\text {bias }}\right)$. The variation of the IR light radiation changes the sensor resistance $\left(R_{\text {sens }}\right)$ and so it does with the voltage drop on the sensor:

$$
\Delta V_{\mathrm{sens}}=I_{\mathrm{bias}} \Delta R_{\mathrm{sens}}
$$

The variation of this voltage drop $\left(\Delta V_{\text {sens }}\right)$ is in fact the signal of interest for the further processing. First, it is restored in terms of both amplitude and frequency by the high-pass pre-amplifier according to the programmable gain $(G)$ and the high-pass cut-off frequency $\left(f_{\mathrm{c}}\right)$, respectively. Then the obtained waveform $\left(V_{\mathrm{amp}}\right)$ is sensed differentially to the equivalent output of the blind channel $\left(V_{\text {blind }}\right)$ by the programmable transconductor $\left(G_{\mathrm{m}}\right)$ to cancel any disturbing signal not related to the IR measurement itself. Finally, the resulting 
effective current signal $\left(I_{\text {adc }}\right)$ is converted into the digital domain by the predictive lock-in A/D converter (ADC). This ADC consists of two stages: a bi-phasic PDM modulator and a digital filter. The IR signal components in the resulting PDM streams (pos and neg) are located in the low-frequency range, while the instantaenous A/D quantification errors are pushed to high frequencies. In this manner and synchronizing the digital lock-in demodulation with the IR emitter signal (lockin), the unwanted components can be easily attenuated by the first-order digital low-pass filter implemented here by an asynchronous 24-bit counter. Almost all the stages of the presented signal processing chain are programmable through a digital configuration register, which is individual for each channel in order to achieve a high level of flexibility, allowing 32768 possible configuration schemes per channel.

\subsection{Pre-Amplification and High-Pass Filtering}

Following the sensor biasing circuit, the first processing stage of the ROIC channel depicted in Fig. 2 simultaneously performs two tasks: the high-pass filtering (DC decoupling) of the IR lock-in pulses and their amplitude amplification. Both of these tasks require a combined active high-pass filtering response. Regarding CMOS integrated circuit design, the key bottle neck for this preamplification stage is the full integration of the low corner lock-in frequencies of the IR thermal sensors (typically below $50 \mathrm{~Hz}$ ) without the use of any external passive component per channel $[8,9]$. To overcome this issue, the MOS-C highpass pre-amplifier topology of Fig. 3 is proposed. Both high-pass filtering and voltage gain are implemented in the present single stage without any external component by using a MOS resistive circuit (MRC) operating in weak inversion (i.e. subthreshold). For high-frequency, the pre-amp behaves as a highly linear capacitive amplifier yielding its gain factor $(G)$ as a ratio between input $\left(C_{\mathrm{A}}\right)$ and feedback $\left(C_{\mathrm{B}}\right)$ capacitors:

$$
G=\frac{\Delta V_{\mathrm{amp}}}{\Delta V_{\mathrm{sens}}}=\frac{C_{\mathrm{A}}}{C_{\mathrm{B}}}
$$




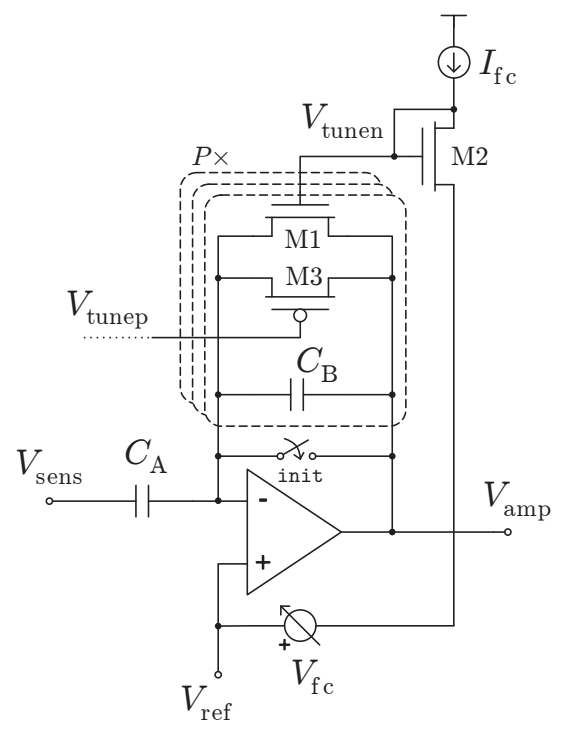

Figure 3: Proposed sub-Hz programmable MOS-C high-pass pre-amplifier. PMOS MRC bias circuit is similar to its NMOS counterpart.

On the other hand, for low-frequency the NMOS feedback transistor M1 and its complementary PMOS transistor M3 supply a feedback path for DC decoupling. A feedback shorting switch is also added for a fast initialization of the pre-amplifier (init high) even for very low corner frequencies. The tuning of the corner frequency itself is automatically obtained by introducing a matched transistor M2, which generates the suitable M1 gate potential ( $\left.V_{\text {tunen }}\right)$ according to the tuning current $\left(I_{\mathrm{fc}}\right)$ and the differential source potential $\left(V_{\mathrm{fc}}\right)$. The generation of the equivalent control $V_{\text {tunep }}$ for M3 is not shown here for simplicity. As M1 and M2 operate in weak inversion conduction and saturation, respectively, the corner frequency of the high-pass response is:

$$
\begin{gathered}
f_{\mathrm{c}}=\frac{1}{2 \pi} \frac{I_{\mathrm{fceff}}}{C_{\mathrm{B}} U_{\mathrm{t}}} \\
I_{\mathrm{fceff}}=I_{\mathrm{fc}} e^{-\frac{V_{\mathrm{fc}}}{U_{\mathrm{t}}}}
\end{gathered}
$$

being $U_{\mathrm{t}}$ and $I_{\text {fceff }}$ the thermal potential and the effective tuning current respectively. Hence, this topology enables a multi-decade $\log$ control of $f_{\mathrm{c}}$ through a compressed voltage range of $V_{\mathrm{fc}}$ (e.g. $f_{\mathrm{c}} \times 10^{ \pm 3}$ requires just $V_{\mathrm{fc}} \pm 173 \mathrm{mV}$ at room 
temperature). Such a $V_{\mathrm{fc}}$ level is internally generated by the programmable circuit proposed in Fig. 4:

$$
\begin{gathered}
V_{\mathrm{fc}}=M U_{\mathrm{t}} \ln (N K) \\
f_{\mathrm{c}}=\frac{f_{\mathrm{co}}}{(N K)^{M}} \text { for } f_{\mathrm{co}}=\frac{1}{2 \pi} \frac{I_{\mathrm{fc}}}{C_{\mathrm{B}} U_{\mathrm{t}}}
\end{gathered}
$$

The corner frequency can be scaled in a wide range even for high $G$ factors i.e. low absolute $C_{\mathrm{B}}$ values in (2). Also, the proposed $f_{\mathrm{c}}$ programming circuit is independent from both temperature and technology variations. In fact, the remaining thermal dependency of $f_{\text {co }}$ in (6) is compensated here using a proportional-to-absolute temperature (PTAT) current reference circuit for $I_{\mathrm{fc}}$ as proposed by these authors in [10]. As validated in Section 5, combining the $V_{\mathrm{fc}}$ tuning with the addition of parallel networks of M1- $C_{\mathrm{B}}$ of Fig. 3, both $f_{\mathrm{c}}$ and $G$ values can be programmed independently.

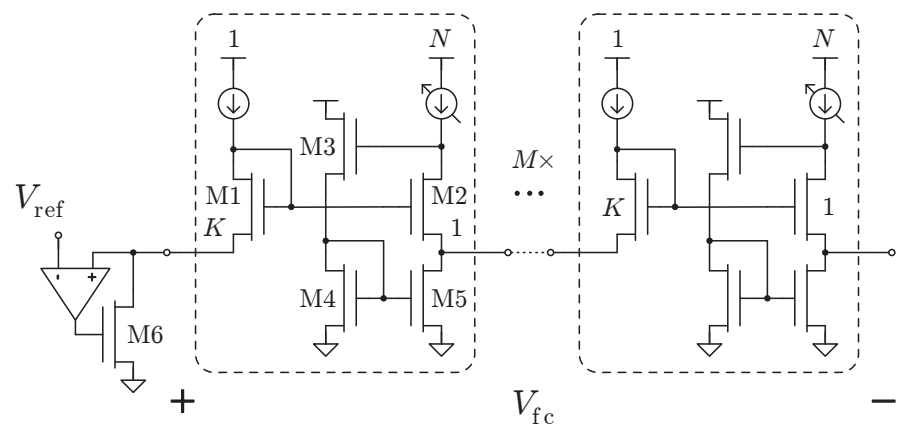

Figure 4: Floating voltage source for the tuning of the MOS-C high-pass pre-amplifier.

\subsection{Blind Cancellation}

Following the pre-amplifying and filtering stage, the second processing stage of the ROIC channel of Fig. 2 is in charge of performing the differential to single-ended amplification of the active pre-amplifier output respect to the blind pre-amplifier reading. Here two operations are performed: cancellation of unwanted disturbing signals generated by thermal, optical, mechanical or even electrical interferences; and $V / I$ conversion of the IR signal to be integrated by the current-mode ADC of the next stage. The transconductance amplifier 
scheme of Fig. 5(a) is implemented for these purposes. The linearization to preserve amplitude information of the lock-in signal is done using the classical cross-coupled differential pair topology M1-M4 operating in strong inversion saturation [11]. Nevertheless, a new additional built-in limiter mechanism is introduced here through M5-M6, which allows programmability of the linear and saturation ranges according to Fig. 5(b). This feature allows to avoid the overload of the ADC stage of next section. M5 and M6 ensure linearity of the cross-coupled structure by keeping a constant current flowing through M2 and M3. When the output signal current reaches $+I_{\max }-I_{\mathrm{gm}}$ (or $\left.-I_{\max }+I_{\mathrm{gm}}\right)$, M5 (or M6) is cut-off and the circuit shows a non-linear compressing curve above (or below) that point. The resulting large signal programmable transconductance can be expressed as:

$$
G_{\mathrm{m}}=\frac{I_{\mathrm{adc}}}{V_{\mathrm{amp}}-V_{\mathrm{blind}}}=2 \sqrt{\frac{2 \beta I_{\mathrm{gm}}}{n}}
$$

being $\beta$ and $n$ the current factor and the subthreshold slope [12] respectively.

\subsection{Pulse Density Modulation}

The scheme of Fig. 6(a) is presented for the 3-level PDM section of the channel ADC of Fig. 2, consisting of a closed loop containing an analog integrator and a window comparator. The analog integrator is based on a previous idea from these authors [13], which exhibits high robustness against reset times. The principle of operation is as follows: during initialization (init high), the analog integrator is reset, while $C_{\text {res }}$ remains connected to $V_{\text {int }}$; once in acquisition (init low), $I_{\text {adc }}$ is integrated in $C_{\text {int }}$ while $C_{\text {res }}$ is tracking the offset, the low frequency noise and the output signal itself of the operational amplifier; finally, when the $\pm V_{\text {th }}$ threshold is reached, the comparator generates a pulse (pos or neg) causing $C_{\text {res }}$ to be switched to the input of the analog integrator. As a result, the charge stored in $C_{\text {int }}$ is compensated by the matched $C_{\text {res }}$ and the reset is completed. It is important to note that this topology does not block the integration of $I_{\text {adc }}$

in $C_{\text {int }}$ during the reset time, behaving like a continuous-time integration during the full acquisition window. Thanks to the absence of dead times during the 


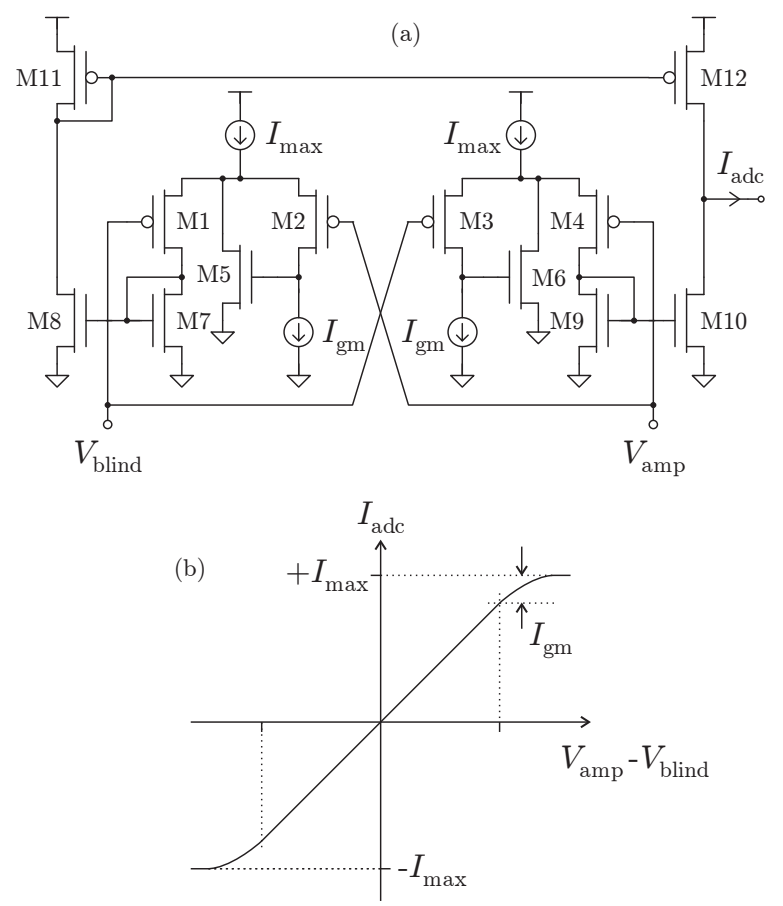

Figure 5: Proposed circuit (a) and linearized transconductance (b) for blind cancellation.

pulse width $\left(T_{\text {pulse }}\right)$ of the PDM stream, as shown in Section 5 , this scheme exhibits a high linear behavior even for output frequency rates $\left(f_{\mathrm{PDM}}\right)$ close to the hard limit of $1 / 2 T_{\text {pulse }}$ :

$$
f_{\mathrm{PDM}}=\frac{I_{\mathrm{adc}}}{C_{\mathrm{int}} V_{\mathrm{th}}}
$$

The window comparator is implemented by a very compact circuit proposed in Fig. 6(b). Instead of generating a dual $\pm V_{\text {th }}$ floating source, an equivalent threshold is built inside the comparators themselves through circuit asymmetries in the M1-M4 cell. In this case, supposing weak inversion saturation for M1-M2:

$$
V_{\mathrm{th}}=n U_{\mathrm{t}} \ln X
$$

The bidirectional pulse counting is performed in the next stage, by the asynchronous counter of Fig. 2, yielding the resulting digital output word at the end 


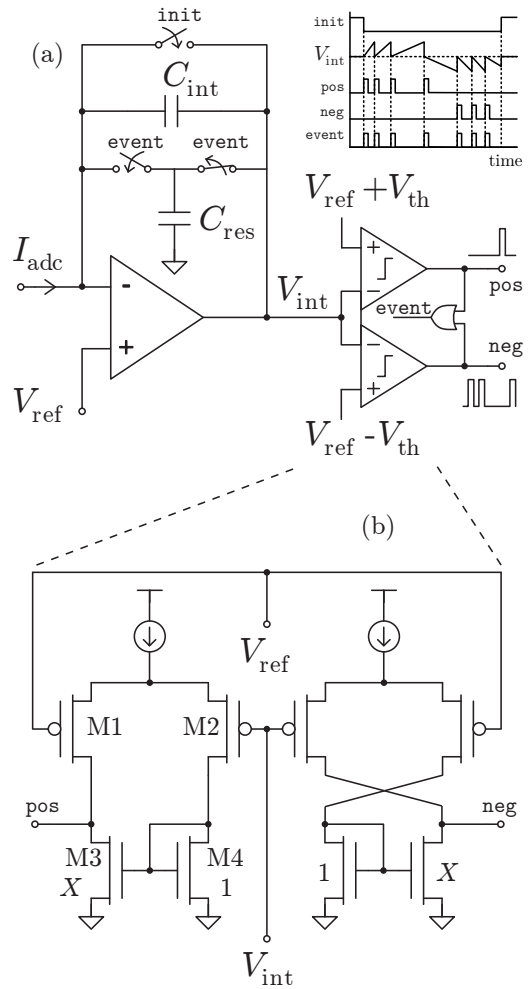

Figure 6: Proposed circuit for the lossless PDM stage of the ADC (a) and detail of the window comparator implementation (b).

of the acquisition window $T_{\text {samp }}$ as:

$$
\begin{aligned}
q_{\mathrm{adc}} & =\left\lfloor n_{\mathrm{adc}}\right\rfloor \\
n_{\mathrm{adc}}=T_{\mathrm{samp}} f_{\mathrm{PDM}} & =\frac{C_{\mathrm{A}}}{C_{\mathrm{B}}} \frac{G_{\mathrm{m}}}{V_{\mathrm{th}}} \frac{T_{\mathrm{samp}}}{C_{\text {int }}} \Delta R_{\mathrm{sens}}
\end{aligned}
$$

\subsection{Digital First-Order Filtering}

In order to overcome issues such as channel crosstalk, clock feedthrough and power wasting, an asynchronous mode of digital low-pass filtering was chosen $[14,15]$. The last stage of the ROIC channel of Fig. 2 is an asynchronous 24-bit counter whose architecture is depicted in Fig. 7. Even though here could be seen a clock input (clk), it is used out of the analog acquisition period $T_{\text {samp }}$ to perform only a digital serial reading of the digital result. The counter consists 
of 22 identical chained counter bit modules following Fig. 8 and two special modules: the least significant bit (LSB) module of Fig. 9 and the most significant bit (MSB) module of Fig. 10. The schematic and layout designs were carried out using a full-custom design techniques, thus decreasing the complexity and occupied area. Each bit of the final resulting word is processed and stored

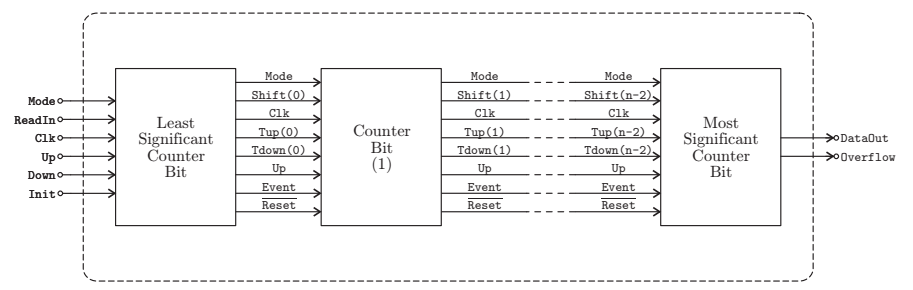

Figure 7: Proposed block scheme for the digital asynchronous low-pass filtering.

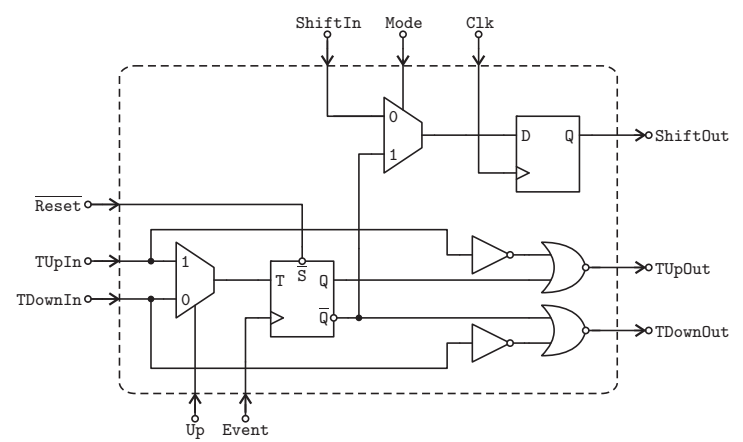

Figure 8: Proposed circuit for the counter bit module of Fig. 7.

by its own counter module. At the beginning of the acquisition cycle, the entire word is initialized (Init high) to a biased value of $2^{23}$ (0x800000). This simplifies the overflow condition detection which coincides with the meeting of the zero condition for both up and down countings. The LSB module starts two toggling complimentary bits (TUpOut and TDownOut) at each positive step event on the counter pulse inputs (Up or Down). A dummy multiplexer and a chain of inverters are used to ensure an appropriate delay and clocking for the following counter bit modules which form a serial connection net between the output-input counting pairs (TupOut-TUpIn, TDownOut-TDownIn). The MSB module has an additional 


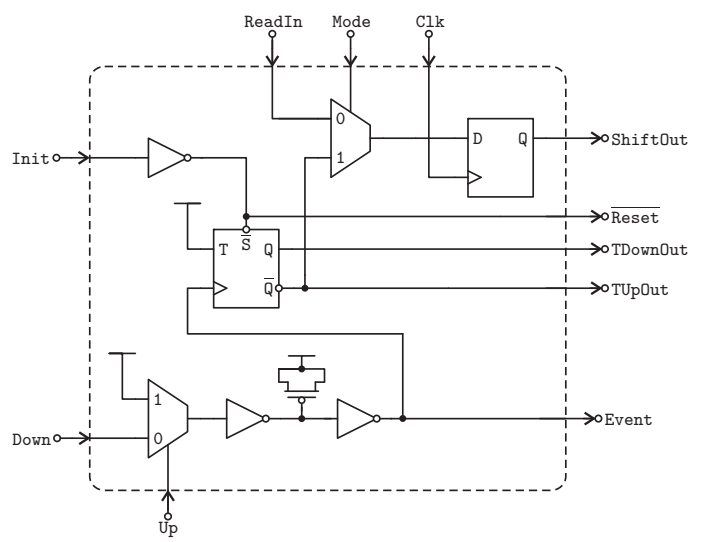

Figure 9: Proposed circuit for the least-significant counter bit module of Fig. 7.

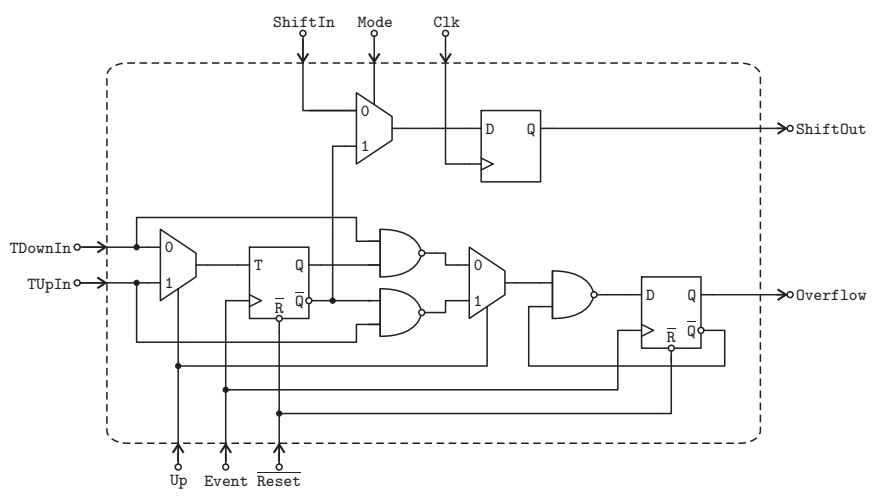

Figure 10: Proposed circuit for the most-significant counter bit module of Fig. 7.

circuitry for the overflow detection, in case of which, the overflow condition flag is activated and remains latched (Overflow high) until the counter initialization is performed.

\section{Post-Layout High-Level Modeling}

As it was mentioned in the previous chapter, the presented ROIC channel has a vast number of possible configurations. In some occasions, it is necessary to carry out a large number of electrical simulations in order to determine the best configuration for different IR sensor types and operating conditions. Due to the complexity of electrical simulation (long simulation time and high CPU 
processing requirements), it is proposed to create a high-level Simulink model for the whole ROIC channel module. In this manner, not only the IC designer, but also the system designer and even the end user are provided with a simple way to preview the ROIC behavior. The complete read-out channel model of

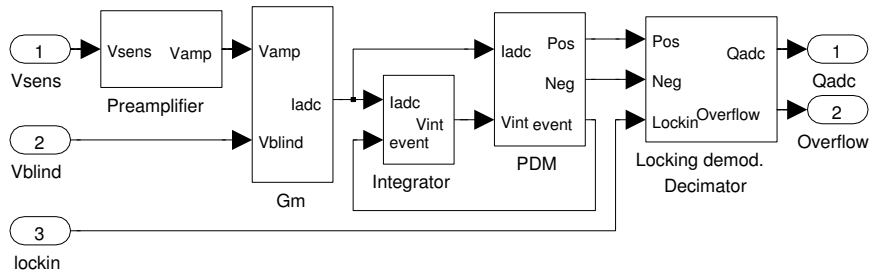

Figure 11: High-level model of the complete read-out channel.

Fig. 11 matches directly its functional description of Fig. 2 with a few exceptions witch are commented below. The first sub-block of the pre-amplifier of Fig. 12 models a high-pass filtering response. The value of the cut-off frequency w_h is read from the table which is loaded before the simulation is started. This table is previously extracted from the electrical simulation done for 12 different cases, combining the programming and worst case sequencing. The same strategy is used for the amplifier inherited low-pass behavior appearing on the higher frequencies. Finally, the noise and DC levels are also generated according to the extracted tables. In the next model stage of Fig. 13 the signals from the

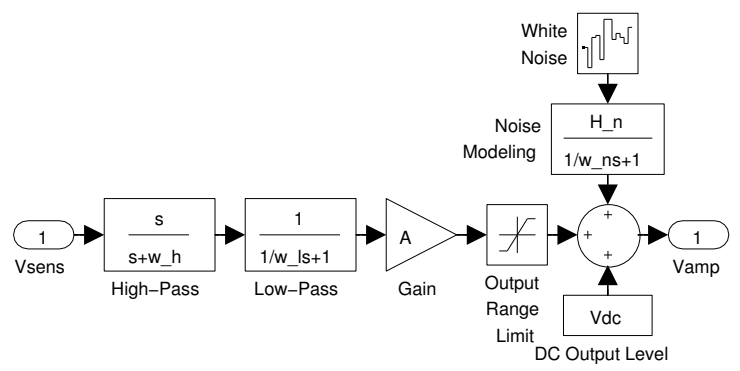

Figure 12: High-pass pre-amplifier model part.

pre-amplifier and blind reference are subtracted (Vamp-Vblind). Then, the same low-pass modeling technique is applied as for the pre-amplifier. The transconductance non-linearity itself is modeled by a look-up table $\mathrm{Gm}$, again considering 
12 simulation cases with 12 different look-up vector arrays. Finally, the noise contributions are added. The extraction of the PDM model is a difficult task

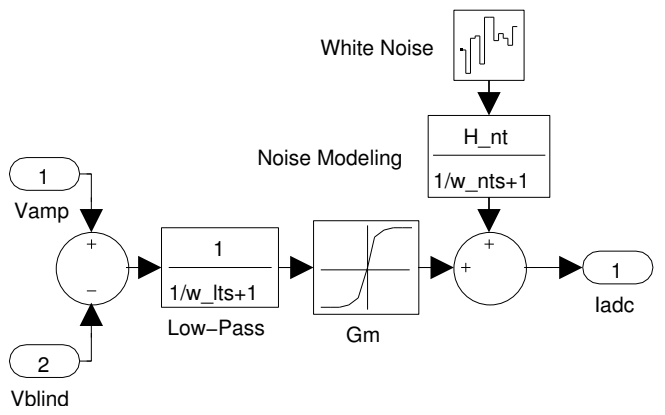

Figure 13: Differential transconductor model part.

due to a large number of parameters and the logarithmic time scaling need for the electrical simulations. The analog integrator model of Fig. 14 and the PDM stage model of Fig. 15 represent the previous proposed circuit of Fig. 6. Depending on the Iadc value, different parameters are corrected through the look-up tables. In general, they determine the voltage ramp generated by the analog integrator and so, the frequency of the Pos and Neg pulses on the output. The pulse duration is determined by the values of the feedback delay read from the initialization table. The last block of the channel model, a digital asynchronous filter of Fig. 16, is done using a bidirectional counter as a trigger sub-block of Fig. 17, which also models the lock-in demodulation before counting. One counting mode is activated if an event occurrs either on the Pos or on the Neg inputs. Depending on which of both pulses is detected, a sum or a subtraction from the qadc word is performed, feeding the result back until the next event is occurred. If a zero condition of counting is detected, the overflow latch is flagged. The presented high level model provides a wide system evaluation flexibility. For instance, in addition to a complete channel programming, it is possible to enable or disable the noise generation for the different blocks together or separately, thus studying the noise contribution for each component. 


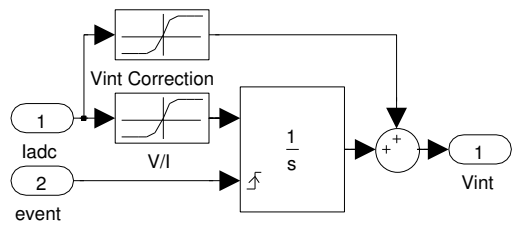

Figure 14: Analog integrator model part.

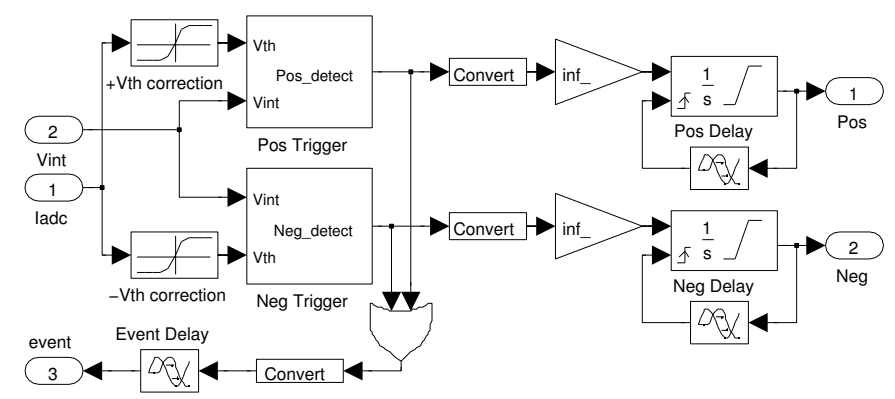

Figure 15: PDM stage model part.

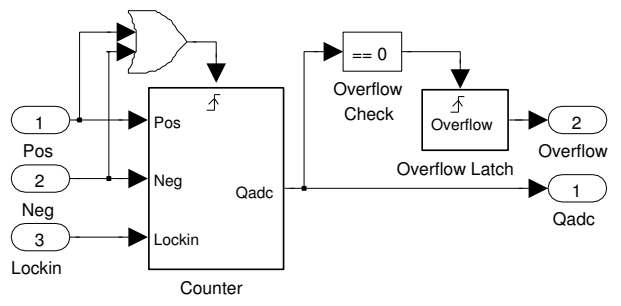

Figure 16: Digital asynchronous filter model part.

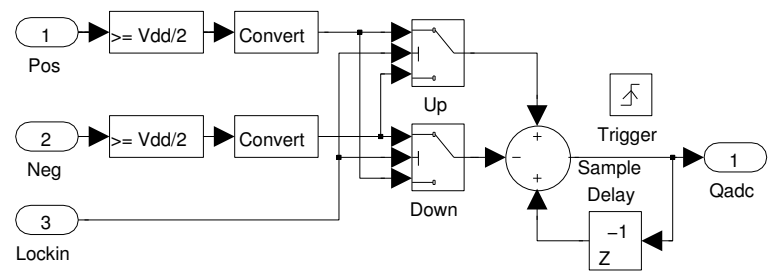

Figure 17: Lock-in demodulation and bidirectional counter model part.

\section{CMOS Integration and Experimental Results}

As shown in Fig. 18, a ROIC full channel has been developed and integrated in $0.35 \mu \mathrm{m}$ CMOS technology based on all the building blocks proposed in the 
previous sections. The most relevant design parameters for this implementation are: $C_{\mathrm{A}}=20 \mathrm{pF}, C_{\mathrm{B}}=\{0.1,0.2,0.4,1\} \mathrm{pF}, K=10, N=\{1,11\}, M=3, I_{\mathrm{fc}}=100 \mathrm{nA}$, $I_{\mathrm{gm}}=3 \mu \mathrm{A}, I_{\mathrm{max}}=8 \mu \mathrm{A}, C_{\mathrm{int}, \mathrm{res}}=\{5,10\} \mathrm{pF}, V_{\mathrm{th}}=120 \mathrm{mV}$ and $T_{\mathrm{pulse}}=725 \mathrm{~ns}$. Table 1 summarizes the rest of programmable parameters and the measured performance. Concerning the overall power consumption, the individual contributions of the pre-amplifier and high-pass filter, the differential transconductor, the pulse density modulator, the digital first-order filter and the rest of the general purpose biasing circuitry vary depending on the channel configuration, but can be averaged as $25 \%, 30 \%, 20 \%, 10 \%$ and $15 \%$, respectively. As seen in Fig. 19, sub-Hz corner frequencies with independent programmability respect to the gain are obtained with up to 16 possible transfer functions (not shown here for simplicity). A dynamic signal analyzer SR785 from Stanford Research Systems was used to perform these sub-Hz measures. The high linearity of the PDM modulator is also verified in Fig. 20, showing no saturation even for stream rates close to the hard limit imposed by the pulse width itself. Although $G_{\mathrm{m}}$ and $C_{\text {int }}$ can not be directly measured, the corresponding $f_{\mathrm{PDM}}$ slopes of Fig. 20 agree with the values obtained from the electrical simulations. Finally, no crosstalk has been observed between the channels.

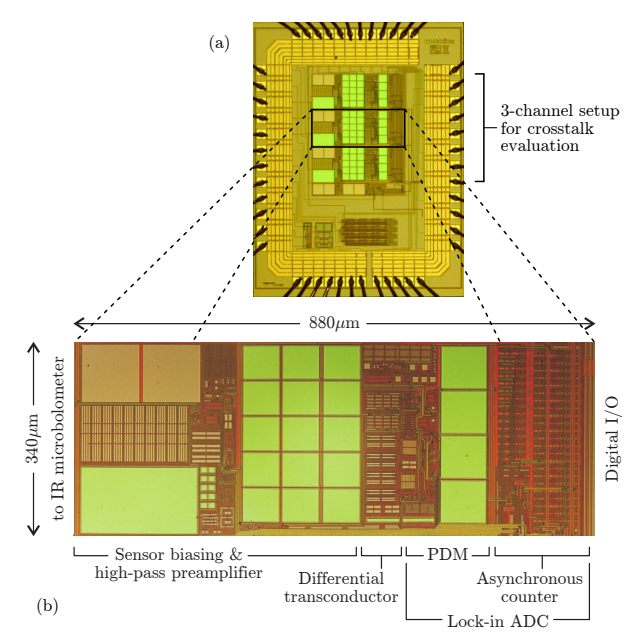

Figure 18: Microscope photography of the test chip (a) and the channel module (b). 

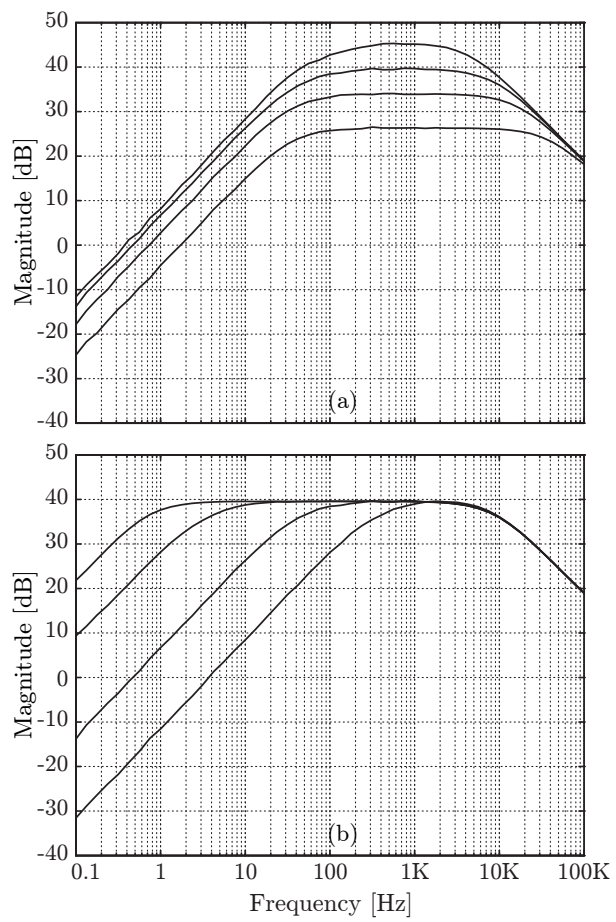

Figure 19: Experimental transfer function of the high-pass pre-amplifier stage for different gain (a) and corner frequency (b) tuning codes.

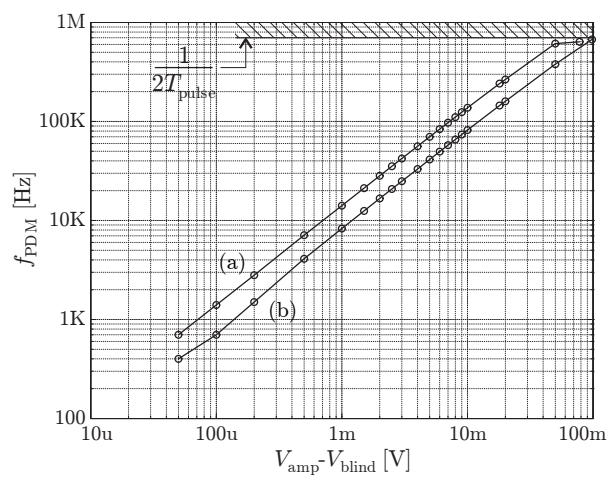

Figure 20: Experimental transfer function of the PDM stage of the predictive ADC for $c$ int $=0$ (a) and cint=1 (b).

\section{Conclusions}

The design and high-level modeling of a low-power, compact and fullyintegrable ROIC channel has been presented for infrared spectroscopic gas recog- 


\begin{tabular}{|c|c|c|}
\hline Parameter & Value & Units \\
\hline$I_{\text {sens }}$ & 1 to 10 & $\mu \mathrm{A}$ \\
\hline$f_{\mathrm{c}}(00)$ & $0.75 \pm 0.10$ & $\mathrm{~Hz}$ \\
\hline (01) & $3.6 \pm 0.4$ & \\
\hline (10) & $49 \pm 8$ & \\
\hline (11) & $389 \pm 76$ & \\
\hline$G(00)$ & $26 \pm 0.1$ & $\mathrm{~dB}$ \\
\hline (01) & $34 \pm 0.1$ & \\
\hline (10) & $40 \pm 0.1$ & \\
\hline (11) & $45 \pm 0.1$ & \\
\hline$G_{\mathrm{m}}(00)$ & 18 & $\mu \mathrm{S}$ \\
\hline (01) & 25 & \\
\hline (10) & 36 & \\
\hline (11) & 45 & \\
\hline $1 / V_{\mathrm{th}} C_{\mathrm{int}}(0)$ & 1.7 & $\mathrm{~Hz} / \mathrm{pA}$ \\
\hline$(1)$ & 0.8 & \\
\hline$V_{\text {sensneq }} @ 10 \mathrm{~Hz}$ & 250 & $\mathrm{nV}_{\mathrm{rms}} / \sqrt{\mathrm{Hz}}$ \\
\hline THD $V_{\mathrm{amp}}<300 \mathrm{mV}_{\mathrm{pp}}$ & $<0.1$ & $\%$ \\
\hline Crosstalk & $<0.5$ & LSB \\
\hline Supply voltage & 3.3 & $\mathrm{~V}$ \\
\hline Supply current & 120 & $\mu \mathrm{A}$ \\
\hline Silicon area & 0.3 & $\mathrm{~mm}^{2}$ \\
\hline
\end{tabular}

Table 1: Experimental results for the frontend channel.

nition. The proposed CMOS circuit includes sensor biasing, sub-Hz high-pass filtering and pre-amplification, blind cancellation, lock-in demodulation, and A/D conversion, all together with independent programmability and thermal compensation. The proposed Simulink high description level channel model allows fast behavioral simulations. The presented experimental results from a $340 \mu \mathrm{m} \times 880 \mu \mathrm{m}$ channel prototype in $0.35 \mu \mathrm{m}$ CMOS technology are in agree- 
ment with the electrical and behavioral simulations. Based on the presented channel module, a 32-channel ROIC is currently under development using the same CMOS technology with a target area of $17.5 \mathrm{~mm}^{2}$ and an overall power consumption of $13 \mathrm{~mW}$.

\section{References}

[1] A. Gore, S. Chakrabartty, S. Pal, E. Alocilja, A multichannel femtoamperesensitivity potentiostat array for biosensing applications, Circuits and Systems I: Regular Papers, IEEE Transactions on 53 (2006) 2357 -2363.

[2] A. Argentieri, F. Corsi, M. Foresta, C. Marzocca, A. D. Guerra, Design and characterization of cmos multichannel front-end electronics for silicon photomultipliers, Nuclear Instruments and Methods in Physics Research Section A: Accelerators, Spectrometers, Detectors and Associated Equipment In Press, Corrected Proof (2010).

[3] A. L. Rosa, G. Mazza, M. Donetti, F. Marchetto, L. Luetto, A. Attili, F. Bourhaleb, R. Cirio, M. Garella, S. Giordanengo, N. Givehchi, S. Iliescu, J. Pardo, A. Pecka, C. Peroni, G. Pittà, Design and test of a 64channel charge measurement asic developed in cmos $0.35 \mu \mathrm{m}$ technology, Nuclear Instruments and Methods in Physics Research Section A: Accelerators, Spectrometers, Detectors and Associated Equipment 583 (2007) 461 -468 .

[4] G. Temes, Micropower data converters: A tutorial, Circuits and Systems II: Express Briefs, IEEE Transactions on 57 (2010) $405-410$.

[5] H. Lin, W. Withayachumnankul, B. M. Fischer, S. P. Mickan, D. Abbott, Gas recognition with terahertz time-domain spectroscopy and reference-free spectrum: A preliminary study, in: Infrared, Millimeter and Terahertz Waves 33rd International Conference, IEEE. 
[6] C. Corsi, N. Liberatore, S. Mengali, A. Mercuri, R. Viola, D. zintu, Advanced Applications to Security of IR Smart Microbolometers, in: Proceedings of the SPIE, volume 6739 of Electro-Optical Remote Sensing, Detection, and Photonic Technologies and thei Applications, pp. 673915-1$673915-10$.

[7] G. Vergara, L. J. Gómez, V. Villamayor, M. Álvarez, M. C. Torquemada, M. T. Rodrigo, M. Verdú, F. J. Sánchez, R. M. Almazán, J. Plaza, P. Rodriguez, I. Catalán, R. Gutierrez, M. T. Montojo, F. Serra-Graells, J. M. Margarit, L. Terés, Monolithic Uncooled IR Detectors of Polycrystalline PbSe: a Real Alternative, in: Proceedings of the SPIE, volume 6542 of Infrared Technology and Applications XXXIII, pp. 20-1-20-9.

[8] A. Tajalli, Y. Leblebici, Power and area efficient mosfet-c filter for very low frequency applications, Analog Integrated Circuits and Signal Processing (2011) 1-10.

[9] A. Tajalli, Y. Leblebici, E. Brauer, Implementing ultra-high-value floating tunable cmos resistors, Electronics Letters 44 (2008) 349 -350.

[10] F. Serra-Graells, J. L. Huertas, Sub-1V CMOS Proportional-to-AbsoluteTemperature References, IEEE Journal of Solid State Circuits 38 (2003) $84-88$.

[11] E. Seevinck, R. F. Wassenaar, A Versatile CMOS Linear Transconductor/Square-Law Funcion Circuit, IEEE Journal of Solid State Circuits 22 (1987) 366-376.

[12] C. C. Enz, F. Krummenacher, E. A. Vittoz, An Analytical MOS Transistor Model Valid in All Regions of Operation and Dedicated to Low-Voltage and Low-Current Applications, Journal of Analog Integrated Circuits and Signal Processing, Kluwer Academic Publishers 8 (1995) 83-114.

[13] J. M. Margarit, J. Sabadell, L. Terés, F. Serra-Graells, A Novel DPS 
Integrator for Fast CMOS Imagers, in: Proceedings of the International Symposium on Circuits and Systems, IEEE, 2008, pp. 1632-1635.

[14] H. Hulgaard, S. M. Burns, G. Borriello, Testing asynchronous circuits: A survey, Integration, the VLSI Journal 19 (1995) 111 - 131.

[15] M. Raji, B. Ghavami, H. Pedram, H. R. Zarandi, Process variation-aware performance analysis of asynchronous circuits, Microelectronics Journal 41 (2010) $99-108$. 